\title{
Ferroelectric domain studies of KNN single crystals by Piezo-force and transmission electron microscopy
}

\author{
Muhammad Asif Rafiq*, Maria Elisabete Costa*, Paula Maria Vilarinho* and Ian M Reaney** \\ * Dep. of Materials and Ceramic Engineering, Centre for Research in Ceramics and Composite \\ Materials, CICECO, University of Aveiro, Campus de Santiago, 3810-193 Aveiro, Portugal \\ ** Department of Materials Science and Engineering Sir Robert Hadfield Building Mappin Street, \\ Sheffield, S1 3JD, United Kingdom
}

Piezoelectric materials find important applications in micro- and nano-electromechanical systems (MEMS/NEMS). $\mathrm{Pb}\left(\mathrm{Zr}_{\mathrm{x}}, \mathrm{Ti}_{1-\mathrm{x}}\right) \mathrm{O}_{3}$ (PZT) is currently the most widely used composition for such applications but due to environmental concerns over the toxicity of lead, lead free alternative materials are required. $\mathrm{K}_{0.5} \mathrm{Na}_{0.5} \mathrm{NbO}_{3}(\mathrm{KNN})$ is considered as a potential lead free piezoelectric [1] but the current generation of monolithic ceramics has inferior electromechanical properties as compared to PZT. Consequently, there is great interest in improving the piezoelectric properties of KNN ceramics and various methods such as doping, hot-pressing and texturing are currently being studied. KNN single crystals like lead based single crystals have shown better electromechanical properties as compared to their ceramic counterparts $[2,3]$. In addition, the behavior of a ferroelectric is largely dependent on its local domain response to an applied electrical or mechanical loading. Therefore, to understand better the material's macroscopic properties, it is essential to access local ferroelectric domains behavior which collectively determines the electromechanical performance.

In this study, piezo-response force microscopy (PFM) and transmission electron microscopy (TEM) were used for imaging the ferroelectric domains in KNN single crystals grown by a high temperature self-flux method. The piezoelectric constant $d_{33}$ was evaluated by a berlincourt piezoelectric meter, sinocera, YE $2730 \mathrm{~A}$. Large KNN single crystals $(4.5 \mathrm{x} 4 \mathrm{~mm})$ were produced by adjusting the various thermal profiles. The used flux was a combination of $\mathrm{K}_{2} \mathrm{CO}_{3}$ and $\mathrm{B}_{2} \mathrm{O}_{3}$ to reduce the meltingtemperature. X-ray powder diffraction of milled crystals, showed the same orthorhombic structure with slight monoclinic distortion as the bulk ceramics (figure 1a), however the single crystals exhibited superior dielectric permittivity especially at high temperature (figure $1 \mathrm{~b}$ ).

Figure 2a presents TEM micrographs of KNN single crystals, in which a distinct domain pattern consisting of bundles of parallel domains can be observed, as encircled and shown in the inset of figure 2a. When the crystals were scanned under PFM, a similar kind of domains pattern was observed in out of phase (OPP) image (figure 2b). The bright and the dark contrast in the image represent the direction of orientation of the polarization vector; dark regions indicate the orientation into the bulk of the crystal (downwards) and the bright regions pointing towards the surface of the crystal (upwards). Analysis of the piezo-signal along the line in figure $2 \mathrm{~b}$ showed positive and negative signal to support this observation. This kind of complex pattern is also found in PZT single crystals [4]. Local hysteresis curve was also measured and it confirmed the domain switching without any sign of leakage behavior as shown in figure 2c. Piezoelectric constant $\left(\mathrm{d}_{33}\right)$ of $160 \mathrm{pC} / \mathrm{N}$ was obtained for single crystals of $\mathrm{KNN}$, which is almost twice as compared to the $90 \mathrm{pC} / \mathrm{N}$ for their bulk counterpart. These results provide a basis for understanding the relationship between the domain patterns and macroscopic properties of KNN single crystal, and should aid the development of optimized KNN as a ferroelectric for practical applications.

\section{References}

1. Y. Saito et al., Nature 432: 84, 2004

2. S. E. Park et al., J. Appl. Phys. 82: 1804, 1997

3. S. Gupta et al., Ferroelect. Lett. 27: 39, 2000

4. V. Anbusathaiah et al., Adv. Mater. 21: 3497, 2009 

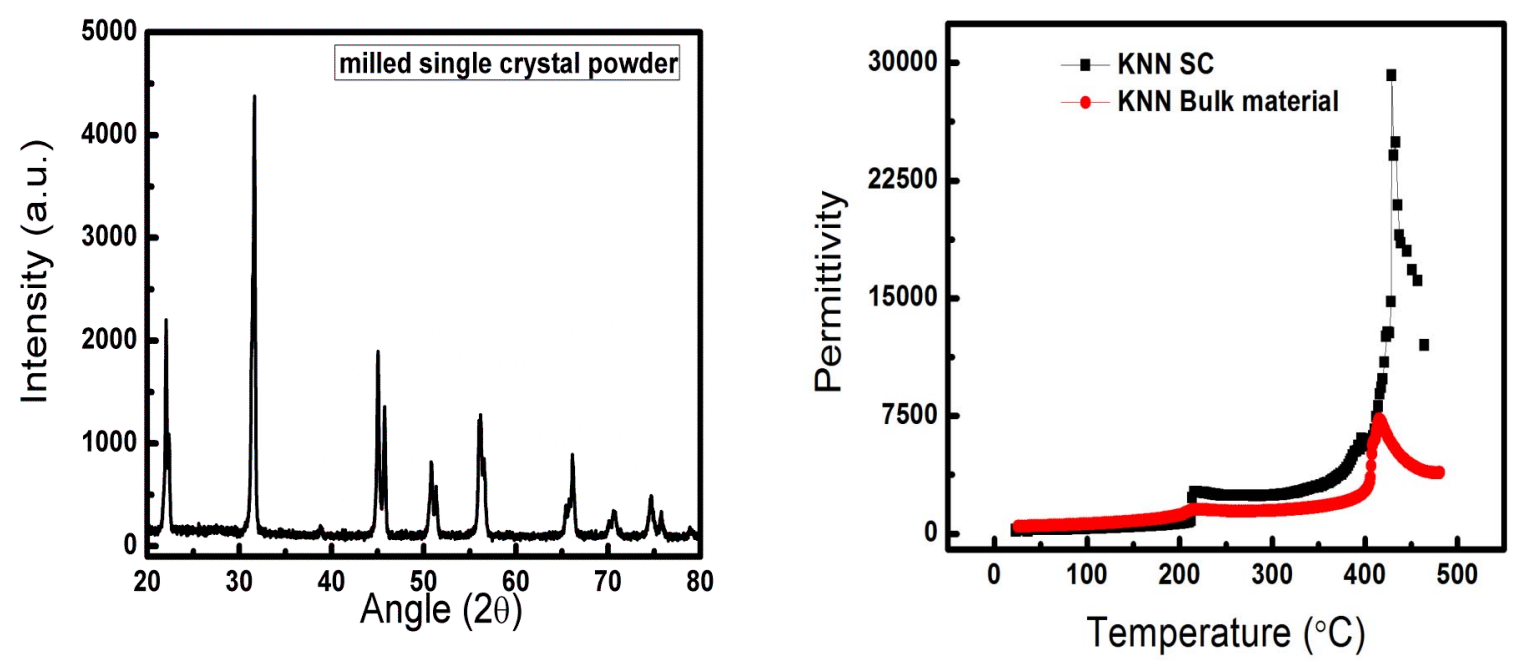

Figure 1. XRD pattern of milled KNN single crystals powder (a) and temperature dependence of permittivity for single crystals and bulk ceramics at $1 \mathrm{kHZ}(\mathrm{b})$.
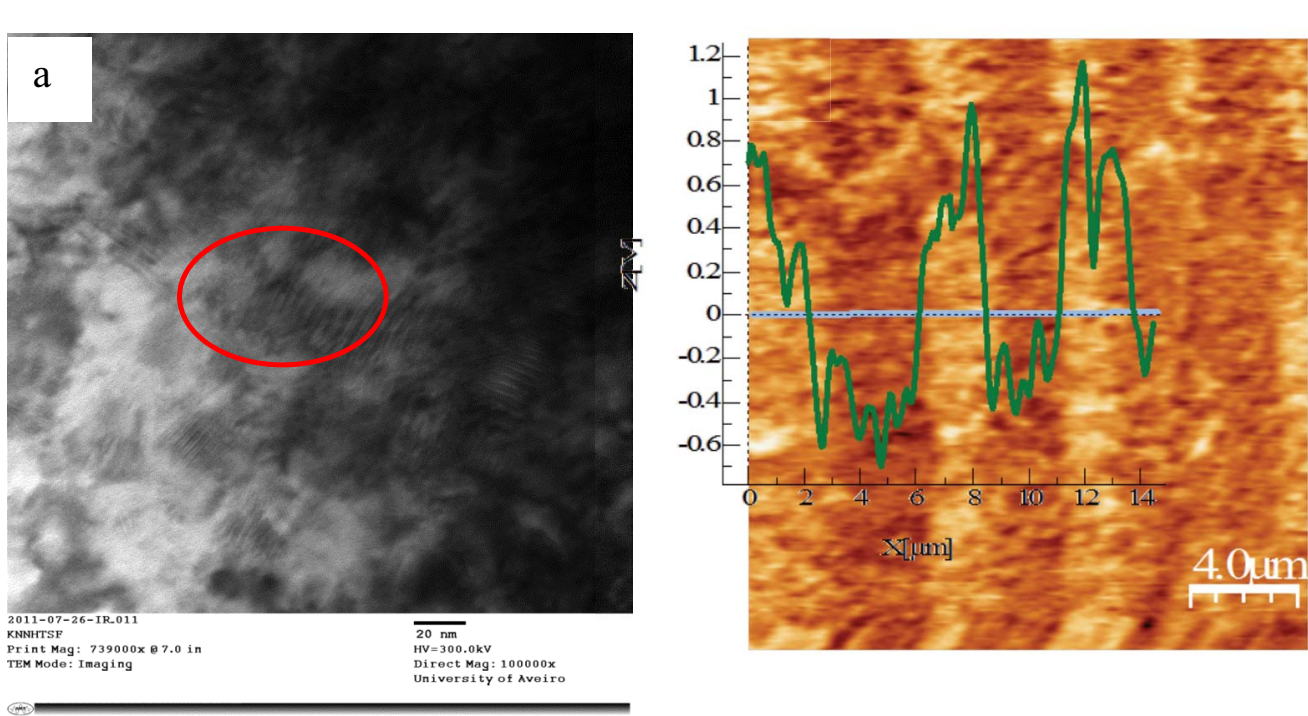

$1.90 \mathrm{~V}$

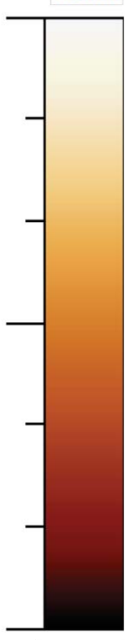

$-1.63 \mathrm{~V}$

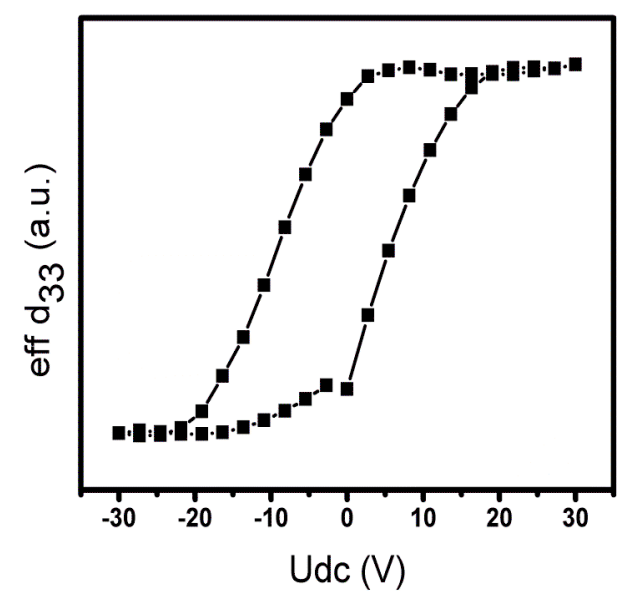

Figure 2. Domains observed by TEM: the inset shows the domains in the zoomed region (a); out of phase image and corresponding piezo- signal of PFM image (b) and local hysteresis curve (c). 